\title{
Observation of Quill Effect induced by Distortion of Spatial Beam Profile
}

\author{
Shigeki Matsuo, Kei Enjo Yoshifumi Umeda, Shuichi Hashimoto \\ Department of Optical Science and Technology, The University of Tokushima \\ matsuo.shigeki@tokushima-u.ac.jp
}

\begin{abstract}
Femtosecond laser modification was carried out for silica substrates using spatially distorted pulses. Modified lines were inscribed in the alternative scanning directions. Etching of the sample revealed significant difference in etching rate, representing Quill effect. Also, the etching rates were affected by the distortion of the spatial beam profile. These results suggest the possibility to control the characteristics of the modified region by controlling the beam profile.
\end{abstract}

\section{Introduction}

One of the important application fields of femtosecond (fs) laser processing is modification inside transparent materials. When a fs pulse was focused inside a transparent material, nonlinear optical phenomena was induced and the material is modified near the focus. This modified region is localized in all three dimensions, thus threedimensional (3D) patterns can be recorded. Optical waveguides were been fabricated inside glasses [1] and other materials. In this case, the focal point was scanned along a line.

Recently, Kazansky and coauthors reported a noteworthy phenomenon, that is, when the scanning direction was reversed, the structure (length of the tracks, angle of periodic structures, and appearance in optical microscopy) of the modified region varied [2]. They called this Quill effect. Later similar phenomena have been reported [3-7]. It should be noted that this is not an effect of polarization, because polarization gives the same effect even if the scanning direction was reversed. We have also reported similar effect by measuring the etching rate along the fs laser-modified lines [8]. Etching in aqueous solution of $\mathrm{KOH}$ was sensitive to the character of the modified region. In this study we used circular polarization, 8 scanning directions were compared.

The origin of the Quill effect must be attributed to the character of the fs laser pulse. It has been proposed that the origin of the Quill effect is pulse front tilt [2], that is, the constant-fluence plane is not perpendicular to the propagation direction, but tilted.

In the present study, we intentionally distorted the spatial beam profile of the fs laser pulse, by inserting a mask in the beam path, so that the shape of focus should also be distorted. Then we observed the occurrence of Quill effect in the etching rate.

\section{Experimental}

The experimental procedure is similar to the previous study [8] except for the application of mask. The mask, made of black aluminum foil, was inserted to the beam path to distort the beam profile. The shape of the mask is shown in Tab. 1. One quarter sector was anisotropically shielded and the energy was reduced to three-fourth of that without mask.

A Ti:Sapphire regenerative amplifier with an operating wavelength of $800 \mathrm{~nm}$ and a repetition rate of $1 \mathrm{kHz}$ was used as the light source. The fs pulses were focused by an objective lens $(40 \mathrm{x}, \mathrm{NA}=0.6)$. The polarization was set to be circular at the focus. During irradiation, the sample (amorphous silica) was scanned alternative directions at a speed of $100 \mu \mathrm{m} / \mathrm{s}$, so that modified lines were fabricated. Totally 6 lines (3 lines in each scanning direction) were fabricated in each irradiation condition (Tab. 1). After irradiation, the sample were cut perpendicular to the lines, and etched in aqueous solution of $\mathrm{KOH}$, and the etching rates were evaluated. The growth of the etched region was in-situ monitored.

Table 1. Irradiation parameters.

\begin{tabular}{|l|c|c|c|}
\hline & (a) & (b) & (c) \\
\hline $\begin{array}{l}\text { Pulse energy } \\
\text { without mask }\end{array}$ & $600 \mathrm{~nJ}$ & $800 \mathrm{~nJ}$ & $800 \mathrm{~nJ}$ \\
\hline Shape of mask & none & & \\
\hline $\begin{array}{l}\text { Pulse energy } \\
\text { with mask }\end{array}$ & $600 \mathrm{~nJ}$ & $600 \mathrm{~nJ}$ & $600 \mathrm{~nJ}$ \\
\hline
\end{tabular}




\section{Results and Discussion}

Figure 1 shows the in-situ images of the sample after etching for about $7.5 \mathrm{~h}$. As seen, the etching rate depended on the irradiation condition and scanning direction. Generally, the lines scanned to right $(\rightarrow)$ etched faster than those scanned to left $(\leftarrow)$, and the difference was significant. In addition, in (b), the etching rate was low; the etched distance was less than half of the others.
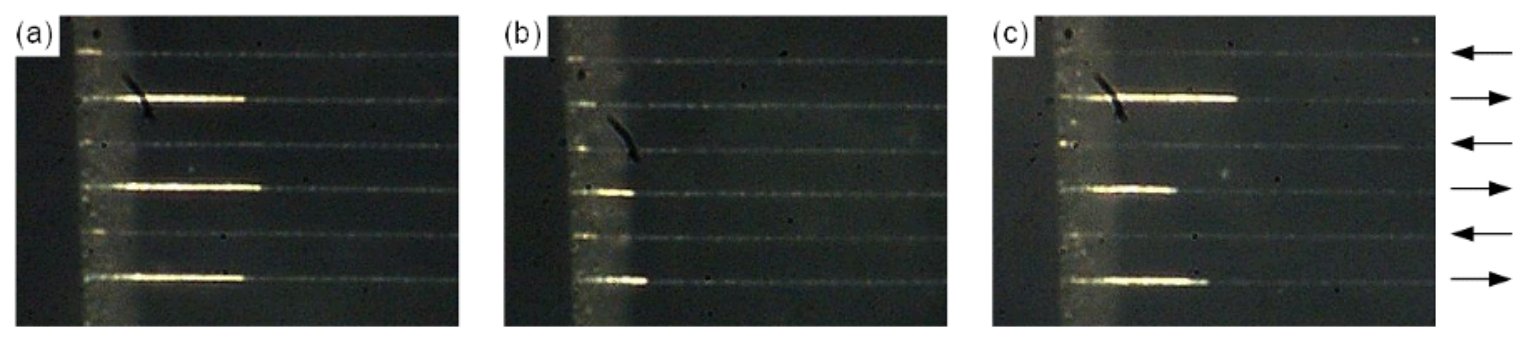

$100 \mu \mathrm{m}$

Fig. 1. Optical microscopy images of modified lines inside the amorphous silica substrates after etching in aqueous $\mathrm{KOH}$ solution $\left(1 \mathrm{M}, 80 \mathrm{C}^{\circ}\right)$ for about $7.5 \mathrm{~h}$. Etching started from edge (left-side in the image), and grew into inside (right) along the modified line. The bright region shows channel structures fabricated. The arrows indicate the direction of laser-scanning during fs laser modification. (a)-(c) corresponds to the irradiation parameters given in Tab. 1.

These results show the existence of Quill effect. The significant difference in etching rate indicates that etching in $\mathrm{KOH}$ solution is a sensitive method to visualize the Quill effect.

In addition, the results shown in Fig. 1 indicate that the spatial beam profile is a parameter which affects to the modification. Maybe this is not surprising, because the shape of beam spot is determined by the beam profile and focusing optics. The standard way is to remove the distortion of beam profile and use a circularly symmetric beam. However, here we suggest that adequate control of the beam profile could be used to control the laser modification including the Quill effect.

It should be noted that Quill effect reported by the other groups used linear polarization [2-7], and most of them used fs laser with a repetition rate of a few hundred $\mathrm{kHz}$ [2-6]. In the present experiments, we used circular polarization and 1-kHz fs laser. Thus, the observed Quill effect could have different characteristic from the previous ones.

\section{References}

[1] K. M. Davis, K. Miura, N. Sugimoto, and K. Hirao, “Writing waveguides in glass with a femtosecond laser,” Optics Letters 21, 1729-1731 (1996).

[2] P. G. Kazansky, W. Yang, E. Bricchi, J. Bovatsek, A. Arai, Y. Shimotsuma, K. Miura, and K. Hirao, “' Quill”' writing with ultrashort light pulses in transparent materials,” Applied Physics Letters, 90, 151120 (2007).

[3] W. Yang, P. G. Kazansky, Y. Shimotsuma, M. Sakakura, K. Miura, and K. Hirao, “Ultrashort-pulse laser calligraphy,” Applied Physics Letters, 93, 171109 (2008).

[4] W. Yang, P. G. Kazansky, and Y. P. Svirko, “Non-reciprocal ultrafast laser writing,” Nature Photonics, vol. 2, 99-104 (2008).

[5] B. Poumellec, M. Lancry, J.-C. Poulin, and S. Ani-Joseph, "Non reciprocal writing and chirality in femtosecond laser irradiated silica," Optics Express, 16, 18354-18361 (2008).

[6] Y. Bellouard and M.-O. Hongler, “Femtosecond-laser generation of self-organized bubble patterns in fused silica,” Optics Express, 19, 6807$6821(2011)$.

[7] D. N. Vitek, E. Block, Y. Bellouard, D. E. Adams, S. Backus, D. Kleinfeld, C. G. Durfee, and J. A. Squier, "Spatio-temporally focused femtosecond laser pulses for nonreciprocal writing in optically transparent materials,” Optics Express, 18, 24673-24678 (2010).

[8] S. Matsuo, Y. Umeda, T. Tomita, and S. Hashimoto, “Laser-Scanning Direction Effect in Femtosecond Laser-Assisted Etching,” Journal of Laser Micro / Nanoengineering, in press (2013). 